\title{
Repurposing new drug candidates and identifying crucial molecules underlying PCOS Pathogenesis Based On Bioinformatics Analysis
}

\author{
Zeinab Dehghan ${ }^{1,2} \cdot$ Samira Mohammadi-Yeganeh $^{2,3} \cdot$ Marzieh Sameni $^{1,2} \cdot$ Seyed Amir Mirmotalebisohi $^{1,2}$. \\ Hakimeh Zali ${ }^{4} \cdot$ Mohammad Salehi ${ }^{2,3}$
}

Received: 7 January 2021 / Accepted: 16 August 2021 / Published online: 4 September 2021

(c) Springer Nature Switzerland AG 2021

\begin{abstract}
Backgrounds Polycystic ovary syndrome affects $7 \%$ of women of reproductive ages. Poor-quality oocytes, along with lower cleavage and implantation rates, reduce fertilization.

Objective This study aimed to determine crucial molecular mechanisms behind PCOS pathogenesis and repurpose new drug candidates interacting with them. To predict a more in-depth insight, we applied a novel bioinformatics approach to analyze interactions between the drug-related and PCOS proteins in PCOS patients.

Methods The newest proteomics data was retrieved from 16 proteomics datasets and was used to construct the PCOS PPI network using Cytoscape. The topological network analysis determined hubs and bottlenecks. The MCODE Plugin was used to identify highly connected regions, and the associations between PCOS clusters and drug-related proteins were evaluated using the Chi-squared/Fisher's exact test. The crucial PPI hub-bottlenecks and the shared molecules (between the PCOS clusters and drug-related proteins) were then investigated for their drug-protein interactions with previously US FDA-approved drugs to predict new drug candidates.

Results The PI3K/AKT pathway was significantly related to one PCOS subnetwork and most drugs (metformin, letrozole, pioglitazone, and spironolactone); moreover, VEGF, EGF, TGFB1, AGT, AMBP, and RBP4 were identified as the shared proteins between the PCOS subnetwork and the drugs. The shared top biochemical pathways between another PCOS subnetwork and rosiglitazone included metabolic pathways, carbon metabolism, and citrate cycle, while the shared proteins included HSPB1, HSPD1, ACO2, TALDO1, VDAC1, and MDH2. We proposed some new candidate medicines for further PCOS treatment investigations, such as copper and zinc compounds, reteplase, alteplase, gliclazide, Etc.

Conclusion Some of the crucial molecules suggested by our model have already been experimentally reported as critical molecules in PCOS pathogenesis. Moreover, some repurposed medications have already shown beneficial effects on infertility treatment. These previous experimental reports confirm our suggestion for investigating our other repurposed drugs (in vitro and in vivo).
\end{abstract}

Keywords Systems biology $\cdot$ Polycystic Ovary Syndrome $\cdot$ Protein-protein interaction network $\cdot$ Infertility $\cdot$ Drug

Mohammad Salehi

m.salehi@sbmu.ac.ir

1 Student Research Committee, Department of Medical Biotechnology, School of Advanced Technologies in Medicine, Shahid Beheshti University of Medical Sciences, Tehran, Iran

2 Cellular \& Molecular Biology Research Center, Shahid Beheshti University of Medical Sciences, Tehran, Iran
3 Department of Medical Biotechnology, School of Advanced Technologies in Medicine, Shahid Beheshti University of Medical Sciences, Tehran, Iran

4 Department of Tissue Engineering and Applied Cell Sciences, School of Advanced Technologies in Medicine, Shahid Beheshti University of Medical Sciences, Tehran, Iran 


\section{Introduction}

Polycystic ovary syndrome (PCOS) is considered a heterogeneous disorder and metabolic dysfunction reported in seven percent of women worldwide in their reproductive ages [1]. Around 45 percent of the infertility cases with unknown causes have PCOS-related morphology associated with ovarian dysfunction or hyperandrogenism. High androgen on PCOS cells affects the incomplete decidual transformation of endometrial cells [2, 3]. Some abnormalities are common in PCOS patients, including (i)-hyperandrogenism, (ii)-polycystic ovary, (iii)-reduced fecundity, (iv) hyperinsulinemia, and (v)-impaired GnRH [4]. It has been reported that some pathways, such as gonadotrophin hormone action, steroid hormone synthesis, and insulin-signaling pathway, play essential roles in PCOS pathogenesis [5-7].

In PCOS patients, treatments are chosen based on specific patient manifestations and are individualized. At present, some drugs have been approved by the US Food and Drug Administration for usage in PCOS. These drugs include metformin, spironolactone, pioglitazone, letrozole, clomiphene, acarbose, and rosiglitazone [8]. These drugs have been reported to be somewhat effective in treating PCOS by affecting oocyte maturation and ovulation abnormalities. The deciphering of their exact pharmacodynamics and pharmacokinetics needs further investigation.

The PCOS mechanism seems to be an enigmatic problem. New genomics and proteomics data, obtained from omics techniques, has brought tremendous information about its molecular pathology, and yet the deciphering of its exact mechanism seems to demand rigorous efforts. Newly, various text-mining strategies and methods have been applied to numerous different molecular biology and medicine tasks, such as drug discovery and molecular study of the disease $[9,10]$. Recently, systems biology has improved our understanding of drugprotein interactions, and different networks of protein-protein interactions (PPI) have been used to predict the mechanisms of drug effects. Several studies have used various PPI networks to decipher underlying molecular mechanisms behind different human conditions [11]. Several others have used PPIs to clarify the molecular mechanisms of responses to drugs [12-15].

More recently, shared proteins among some associated diseases have been used to deepen our understanding of their molecular pathology in more detail [11]. Shared proteins between drug-related protein networks and disease PPI networks can help obtain new insights into the disease's molecular pathology. It helps elucidate the essential proteins, which can probably be used to prevent, diagnose, and drug design.

In this in-silico study, we pursue two different goals. We will first analyze the PCOS protein-protein interactions (PPI) to identify the PCOS pathogenesis's essential molecular mechanisms. We will then determine the significant interactions between the drug-related proteins (textmined) and the PCOS-related protein subnetworks. Using this method will probably help us gain a more in-depth insight into the PCOS molecular mechanisms using the novel in-silico approach. Second, we will repurpose new drug candidates for PCOS treatment for further experimental studies since most current infertility medications are symptom-based, and investigations for better treatments coping with PCOS metabolic and reproductive abnormalities seem necessary [16]. In this study, we will first gather data on proteins related to PCOS to achieve the goals. We also will collect information on drugs involved in ovulation. We will then construct the PPI networks of interactions among the PCOS-related proteins and drug-related proteins to deepen our understanding of the PCOS molecular mechanism. After determining the crucial molecules using the analysis of PCOS PPI and PCOS drug-related protein networks, we will repurpose new drug candidates using the identified essential molecules.

\section{Methods}

\section{Design of the study}

Recently bioinformatics approaches have been used to predict the molecular mechanisms behind some reproductive diseases [28, 29]. This study has used a novel bioinformatics approach to investigate the relations between the infertility drug-related proteins and the PCOS PPIN. The graphical workflow is represented in Fig. 1.

\section{Data collection}

In the current study, we extracted data from 16 proteomics papers available in PCOSBase (Supplementary Table S1). PCOSBase is a manually curated medical database. It is compiled from different genes and protein expression research papers and nine databases (including Disease [10], GWAS Catalog [17], MalaCards [18], GWASdb [19], Online Mendelian Inheritance in Man (OMIM) [20], Disease and Gene Annotation (DGA) [21], PhenomicDB [22], DisGeNet [23], and the Human Gene Mutation Database (HGMD) [24]). In sum, we obtained 168 up and down-regulated proteins in PCOS patients compared to the normal (Table S1). These studies have identified the differences in the amount of protein using mass spectroscopy, MALDI-TOF-MS, or LC-MS techniques. The investigated tissues included the ovary, granulosa cells, and follicular fluid.

Due to the significant role of the oocyte maturation and ovulation abnormalities in PCOS pathology, we selected 
Fig. 1 Workflow. A graphical overview of the workflow

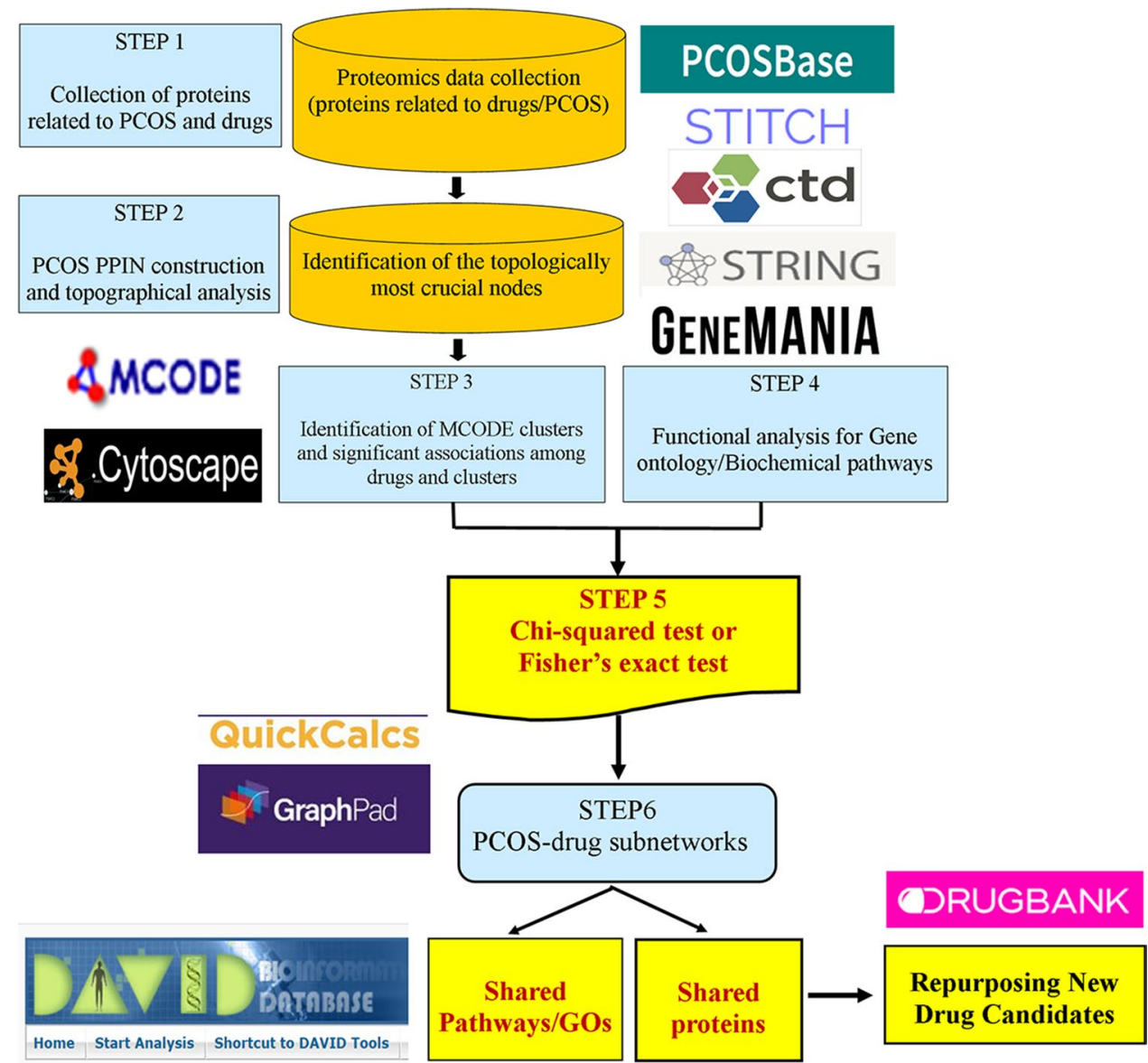

the clinical drugs used to treat infertility in PCOS that target these abnormalities (according to the literature). These drugs included acarbose, clomiphene, letrozole, metformin, pioglitazone, rosiglitazone, and spironolactone [8]. The proteins related to these drugs (experimental textmined relation) were then obtained using two experimental databases (including CTD and STITCH) (Table S2). The comparative Toxicogenomics Database (CTD) reports the experimented text-mined relations among diseases, drugs, genes, chemicals, environmental exposures, and phenotypes [25]. The STITCH database also represents the different types of interactions between drugs and proteins [26].

\section{Construction of PCOS PPI Network}

PCOS Protein-protein interaction network (PPI) was constructed using the Protein-protein interaction map available in the STRING database (https://string-db.org/). STRING is a database that uses experimental data and computational prediction methods to predict protein-protein interactions. We used Cytoscape 3.5.1 software to visualize the PPI network and further analyses [40].

\section{Topological analysis and functional enrichment of hubs}

Cytoscape Network Analyzer was used to analyze the topological parameters, including Degree and Betweenness Centrality (BC). Nodes with higher degree values were considered as the hubs. Nodes with higher Betweenness Centrality were considered as bottlenecks [38]. Functional analysis was then performed on the top ten hub nodes using GeneMANIA. GeneMANIA is a tool for gene function prediction [41].

\section{Construction of PPI subnetworks}

The Molecular Complex Detection (MCODE) app was used to determine the highly interconnected PCOS network regions, called MCODE clusters. The MCODE identifies the densely connected subnetworks from a vast interaction network. Subnetworks with a score of interaction higher than 2.0 and at least two nodes were identified as significant. (The MCODE parameters settings included Degree Cutoff $=2$, Node Score Cutoff $=0.2$, K-Core $=2$, and MaxDepth $=100$.) [27]. 
The number of shared proteins between the PCOS PPI subnetworks and drug-related proteins was then investigated to determine the significant relations between different MCODE motifs and drugs. The chi-squared test or Fisher's exact test (taking into account the requirements of the Chi-square test) were used to identify statistically available significant relations between the drugs and the subnetworks obtained from the PCOS PPI network ( $p$-value $<0.05)$. The chi-squared test only works for random data and independent samples, and its sample size must be large enough [28]. Fisher's exact test was used when the chi-square conditions were not met. The $2 \times 2$ contingency tables have been used to analyze the statistically significant relations using the GraphPad online calculator [29]. This method can be used to evaluate the associations between diseases or assess the significance of a relationship between disease and their disease-associated genes [11, 30, 31]. The associations were evaluated using $\mathrm{a}, \mathrm{b}, \mathrm{c}$, and d numbers shown in Table 1 .

The PCOS subnetworks significantly related to the PCOS drugs were then classified as PCOS-drug subnetworks and were selected for the biochemical pathway and biological process enrichment analysis to identify shared pathways and processes between PCOS and the drugs.

Table $12 \times 2$ contingency table. Fisher's Exact test with values: a, b, $\mathrm{c}$, and d

\begin{tabular}{llll}
\hline & $\begin{array}{l}\text { Shared between } \\
\text { the drug and the } \\
\text { PCOS-PPI network }\end{array}$ & $\begin{array}{l}\text { Non-shared } \\
\text { between the drug } \\
\text { and PCOS-PPI } \\
\text { network }\end{array}$ & \\
\hline In subnetwork & $\mathrm{a}$ & $\mathrm{b}$ & $\mathrm{a}+\mathrm{b}$ \\
Outside subnetwork & $\mathrm{c}$ & $\mathrm{d}$ & $\mathrm{c}+\mathrm{d}$ \\
& $\mathrm{a}+\mathrm{c}$ & $\mathrm{b}+\mathrm{d}$ & $\mathrm{n}$ \\
\hline
\end{tabular}

Note: $n^{*}$ was the number of all proteins available in the PCOS-related PPI network

\section{Biochemical Pathway and biological process enrichment analysis}

Biochemical pathway and biological process enrichment analysis were carried out using the Database for Annotation, Visualization, and Integrated Discovery (DAVID) (https:// david.ncifcrf.gov/) [32] according to the Kyoto Encyclopedia of Genes and Genomes (KEGG) database [33]. DAVID provides functional annotation tools for analyzing biological themes for lists of genes [32]. This database was used to identify pathways and biological processes shared between subnetworks and drugs. Shared Biochemical Pathways were determined between the medicines and PCOS subnetworks.

\section{Drug-protein interaction analysis}

We constructed a collection of proteins containing two main groups. First, we selected the most crucial molecules of the PPI (hub-bottlenecks) (Table 2). Second, we added the shared molecules between PCOS MCODE clusters and the drug-related proteins to the collection (Table 3 ). We then used the DrugBank database (https://go.drugbank.com/) to repurpose new drug candidates for the crucial proteins. The new recommended drugs were selected from the previously approved drugs readily available in DrugBank. Finally, we visualized the interaction network between new repurposed drugs and the selected proteins using Cytoscape 3.5.1 software.

\section{Results}

\section{PCOS Protein-protein interaction network}

The number of differentially expressed proteins in PCOS patients was 168. (107 up-regulated proteins and 61 downregulated proteins). The protein network was constructed and analyzed using the STRING database and Cytoscape
Table 2 The List of 10 top hub proteins with the highest degrees

\begin{tabular}{lllll}
\hline $\begin{array}{l}\text { The human-read- } \\
\text { able label name }\end{array}$ & Description & Uniprot & Degree & Betweenness Centrality \\
\hline ALB & Serum albumin & P02768 & 55 & 0.1523043 \\
FN1 & Fibronectin & P02751 & 49 & 0.08806922 \\
VEGFA & Vascular endothelial growth factor-A & P15692 & 38 & 0.06025576 \\
FGA & Fibrinogen alpha chain & P02671 & 35 & 0.02468968 \\
EGF & Epidermal growth factor & P01133 & 35 & 0.06421301 \\
PLG & Plasminogen & P00747 & 32 & 0.02962186 \\
FGG & Fibrinogen gamma chain & P02679 & 31 & 0.01531035 \\
KNG1 & Kininogen-1 & P01042 & 31 & 0.03993729 \\
P4HB & Protein disulfide isomerase & P07237 & 30 & 0.05458324 \\
APOA1 & Apo lipoprotein A-I & P02647 & 30 & 0.02361636 \\
\hline
\end{tabular}


Table 3 The PCOS-drug subnetworks. The table represents the shared proteins between MCODE subnetworks and drugs in which the relationships were statistically significant

\begin{tabular}{|c|c|c|c|c|c|}
\hline $\begin{array}{l}\text { PCOS subnet- } \\
\text { work }\end{array}$ & Density score & $\begin{array}{l}\text { Number of } \\
\text { Nodes }\end{array}$ & $\begin{array}{l}\text { Number of Inter- } \\
\text { actions }\end{array}$ & Associated drugs & Shared proteins \\
\hline No. 2 & 7.75 & 17 & 62 & $\begin{array}{l}\text { Spironolactone } \\
\text { Pioglitazone } \\
\text { Metformin } \\
\text { Letrozole }\end{array}$ & $\begin{array}{l}\text { VEGF-A, TGFB1, AGT } \\
\text { AGT, EGF, VEGFA, TGFB1 } \\
\text { AMBP, RBP4, AGT, VEGFA, TGFB1 } \\
\text { VEGF-A, TGFB1 }\end{array}$ \\
\hline No. 3 & 6.353 & 18 & 54 & Rosiglitazone & $\begin{array}{l}\text { HSPB1, HSPD1, ACO2, TALDO1, } \\
\text { VDAC1, MDH2, HSPA5, ATP5J, } \\
\text { BCL2L1, PKM }\end{array}$ \\
\hline
\end{tabular}

3.5.1 software. The PPI network from the "significantly altered PCOS proteins was then analyzed using the Cytoscape Network Analyzer tool to identify hubs and bottlenecks [34]. Table 2 represents ten top hub proteins with the highest degrees. Figure 2 depicts the PCOS PPI network, and the hub nodes are shown with a bigger size, and their colors are closer to dark red color. The top ten hubs were functionally enriched using GeneMANIA, and the results are shown in Supplementary Fig. S1. Each hub node is depicted with various colors in the supplementary

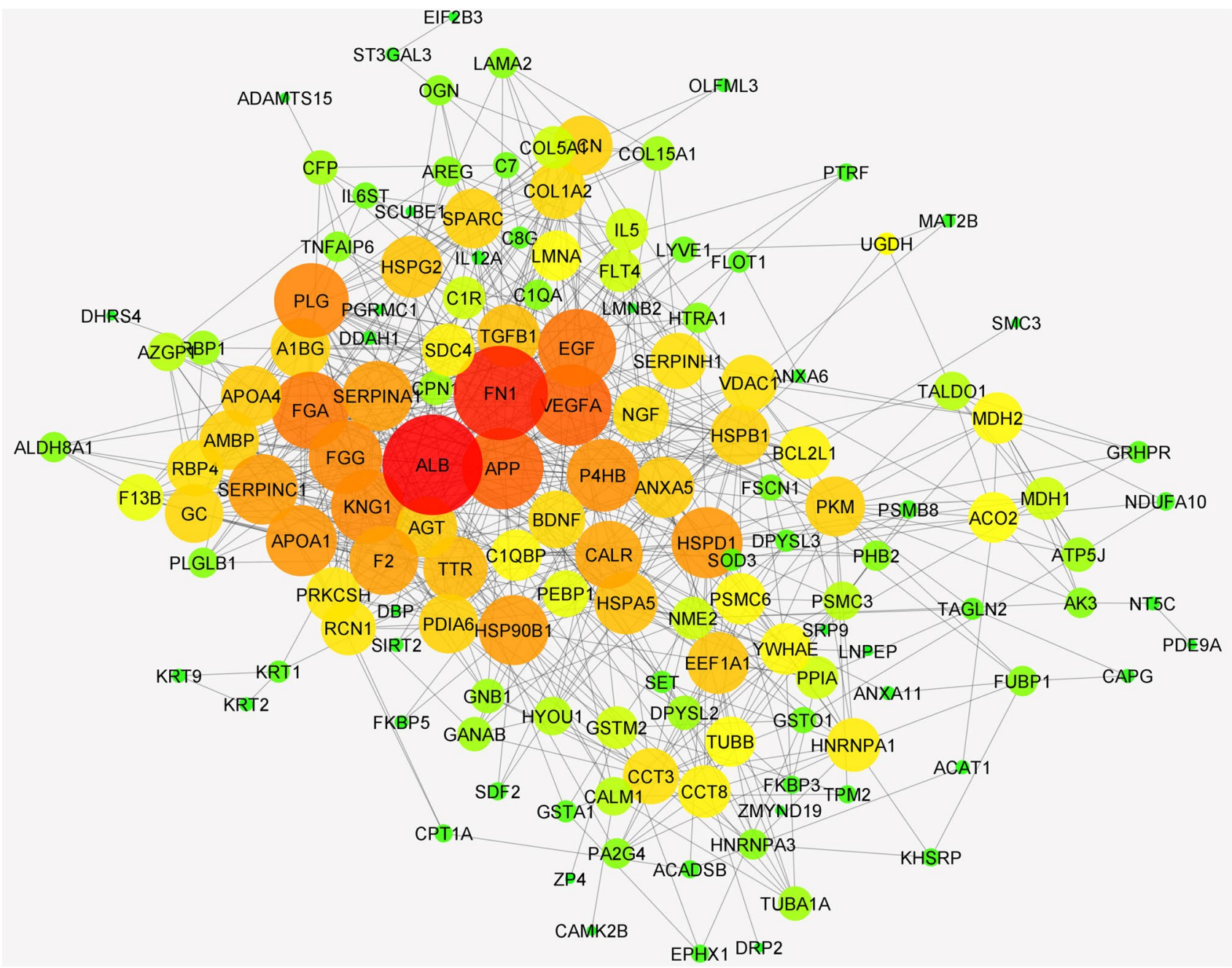

Fig. 2 PCOS PPI network. The PCOS PPI network is visualized using Cytoscape 3.5.1 software. The top ten hubs (nodes with a higher degree) are shown with a bigger size, and their color is closer to dark red 
figure, and the different colors represent different biological functions.

\section{PCOS PPI MCODE clusters}

The MCODE plugin identified five subnetworks as significant in the PCOS PPI network. (The MCODE parameters settings included Degree Cutoff $=2$, Node Score Cutoff $=0.2$, K-Core $=2$, and Max-Depth $=100($ (Table S3). Figure 3 depicts the highly connected regions (clusters) identified using the MCODE app.

\section{PCOS-drug subnetwork}

After analyzing the data using the chi-squared test or Fisher's exact test (based on prerequisites), we found that five drugs had significant relations with two of the five PPI subnetworks (subnetworks No.2 and No.3, $p$-value $<0.05$ ). (Supplementary Table S4) These five drugs included metformin, letrozole, pioglitazone, spironolactone, and rosiglitazone. Subnetwork No.2 showed a significant relationship with pioglitazone, metformin, spironolactone, and letrozole. We found that subnetwork No.3 was significantly associated with rosiglitazone. The results and shared proteins between subnetworks and drugs are available in Table 3 and Table S5.

Three proteins of subnetwork No.2 were found to be shared with spironolactone-related proteins. These included VEGFA (Vascular endothelial growth factor), TGFB1 (Transforming Growth Factor beta-1), and AGT (Angiotensin). PCOS showed association with pioglitazone in subnetwork No.2 with four shared proteins, including VEGFA, TGFB1, AGT, and EGF (Epidermal growth factor). Subnetwork No.2 also highlighted the relation of PCOS with metformin. The VEGFA, TGFB1, AGT, AMBP (Alpha-1-Microglobulin/Bikunin Precursor), and RBP4 (Retinol binding protein 4) were shared proteins between PCOS subnetwork No.2 and metformin. Besides, letrozole was accompanied by Subnetwork No.2 and shared some proteins, including VEGFA and TGFB1. Interestingly, the results showed that VEGFA and TGFB1 were the essential shared proteins among subnetwork No.2 and all the four drugs' proteins.

Subnetwork No.3 showed a significant relation with rosiglitazone due to ten shared proteins, including HSPB1

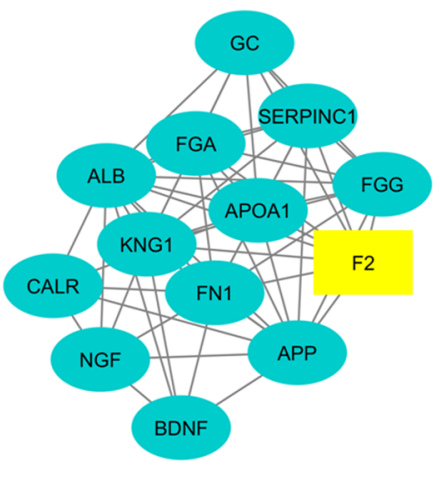

Score: 9.5

Nodes: 13

Edges: 57

Seed: F2

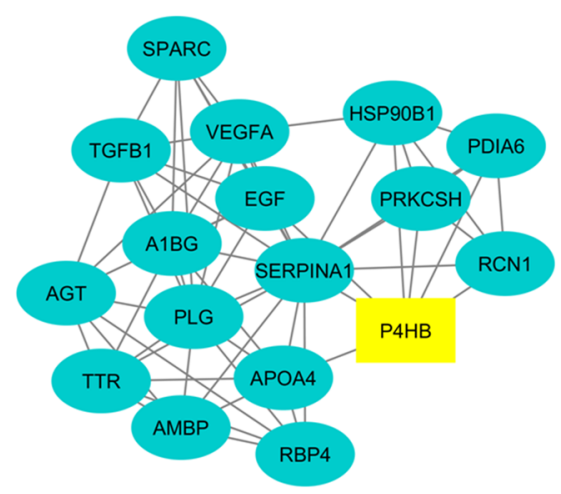

Score: 7.75

Nodes: 17

Edges: 62

Seed: P4HB

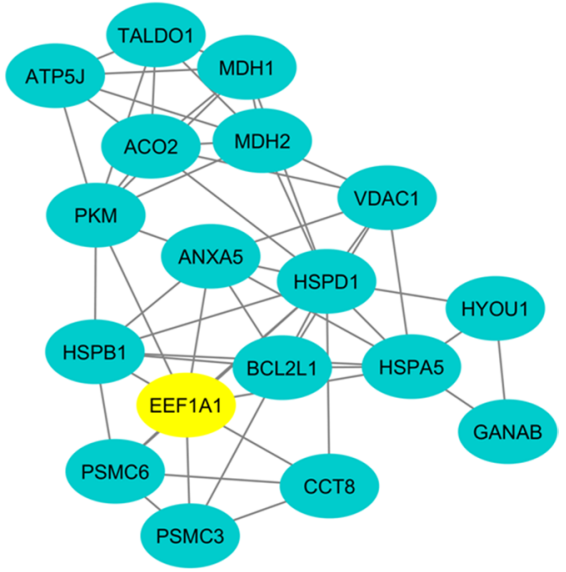

Score: 6.35

Nodes: 18

Edges: 54

Seed: F2

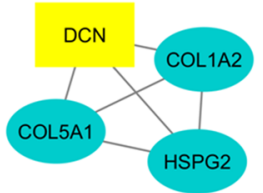

Score: 4

Nodes: 4

Edges: 6

Seed: DCN

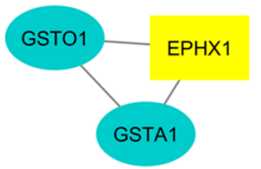

Score: 3

Nodes: 3

Edges: 3

Seed: EPHX1

Fig. 3 The figure represents the MCODE clusters. The PPI subnetworks (clusters) are created using the MCODE app from highly connected regions of the PCOS PPI network. The yellow nodes represent the seeds 
(Heat Shock Protein Family B (Small) Member 1), HSPD1 (Heat Shock $60 \mathrm{kDa}$ Protein 1), ACO2 (Aconitase 2), TALDO1 (Transaldolase 1), VDAC1 (Voltage-dependent anion-selective channel 1), MDH2 (Malate Dehydrogenase 2), HSPA5 (heat shock $70 \mathrm{kDa}$ protein 5), ATP5J (ATP synthesize-coupling factor 6), BCL2L1 (Bcl-2-like protein 1), and PKM (Pyruvate kinase).

\section{Biochemical pathway and biological process enrichment analysis}

The biochemical pathways involved in both PCOS subnetworks (NO.2, NO.3) and the five drugs were analyzed using pathway enrichment analysis by the DAVID tool (Tables S6, S7). The shared pathways between PCOS subnetworks and the drugs were then investigated (Table S8). Interestingly, the PI3K-Akt signaling pathway was a significant pathway between subnetwork No." 2 and the four results of drugrelated protein enrichment (pioglitazone, spironolactone, letrozole, and metformin). VEGFA and EGF were found to be the two shared proteins in the PCOS-drug network. They were also both involved in the PI3K-Akt signaling pathway. (Fig. 4).

Six biochemical pathway terms identified as significantly enriched terms in both subnetwork No.3 and rosiglitazone included 1-metabolic pathways, 2-Carbon

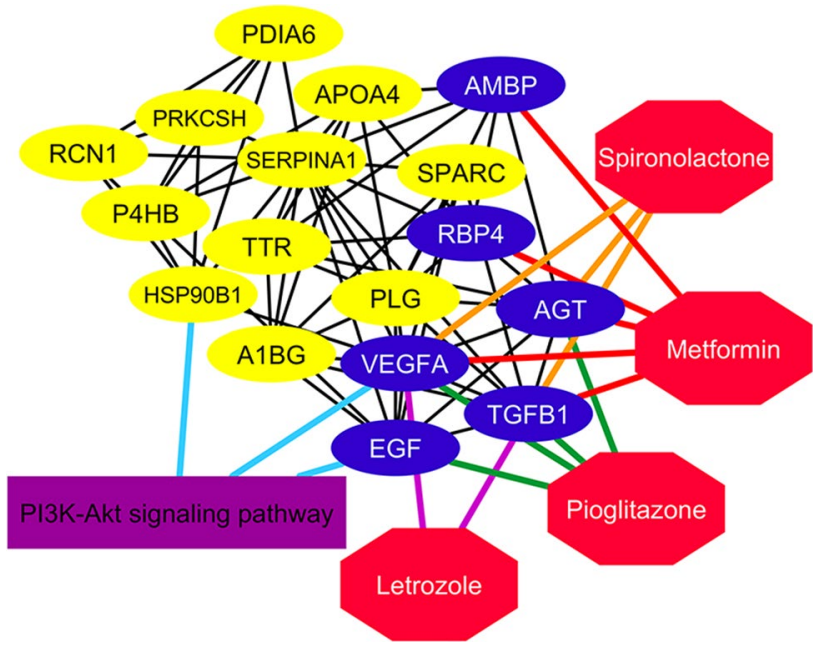

Fig. 4 The figure represents the interactions among the four drugs and PCOS subnetwork No.2. The ellipses show the PCOS-related proteins, octagons represent the drugs, and the squares depict pathways. The shared proteins between PCOS subnetwork No.2 and the four drugs (Metformin, Pioglitazone, Letrozole, and Spironolactone) are red. PCOS-drugs interactions are shown by edges in four colors, including red for metformin, orange for spironolactone, green for pioglitazone, and violet for letrozole. The light blue lines indicate the interactions of the PI3K-AKT pathway. Yellow nodes and black edges indicate the PCOS-related proteins' interactions in subnetwork No.2 that are not shared with drug-related proteins metabolism, 3-citrate cycle (TCA cycle), 4-pyruvate metabolism, 5-biosynthesis of amino acids, and 6-glyoxylate/dicarboxylate metabolism. The shared proteins between subnetwork No.3 and rosiglitazone included: HSPB1, HSPD1, ACO2, TALDO1, VDAC1, MDH2, HSPA5, ATP5J, BCL2L1, and PKM. The shared pathways and proteins between subnetwork No.3 and rosiglitazone are shown in Fig. 5. Table 4 represents the shared pathways significantly enriched for the PCOS-drug subnetworks. The shared biochemical pathways related to the ovaries, encompassing the shared genes, are given in Table 4. (Other results are available in Table S8).

The biological processes intermediating in both subnetworks (NO.2, NO.3) and the five drugs were analyzed using the GO enrichment analysis by the DAVID tool (Tables S9, S10). The results showed that the shared proteins between PCOS subnetworks and drugs participated in various biological processes. They were involved in different vital biological processes, including 1-secretion, 2-response to stress, 3-regulated exocytosis, and 4-platelet degranulation. Table No.5 represents the shared biological processes between enrichment results of the drug-related proteins and PCOS cluster proteins. The top three shared biological process terms between each drug and subnetwork and their shared genes are represented in Table 5. (The other results are available in Table S11; ranked base on $P$-value(

\section{Drug-protein interaction analysis}

We evaluated the twenty proteins for their drug-protein interactions with previously US FDA-approved drugs available in DrugBank. DrugBanak is a database that has provided information on protein-drug direct and indirect interactions. Our selected target proteins included the PPI hub-bottlenecks and the shared molecules between PCOS clusters and drug-related proteins (our new repurposed drugs are represented in Table 6). Figure No.6 depicts the drugprotein interactions (direct and indirect) visualized using Cytoscape. For example, copper interacted with eight proteins (PKM, PLG, AGT, APOA1, KNG1, P4HB, HSPD1, HSPA5). Zinc chloride and zinc sulfate had interactions with FN1, P4HB, APOA1, KNG1, FGA proteins. Moreover, zinc and zinc acetate interacted with FN1, P4HB, APOA1, KNG1, FGA, AGT proteins. Reteplase and alteplase were two other drugs interacting with FGA and PLG proteins. VEGF and ALB proteins were related to a medicine named gliclazide. Hyaluronidase (ovine) was linked to ALB and TGFB1. Figure 6 depicted the drug-protein interaction network. Supplementary Table S11 represents the repurposed US FDA-approved medications for PCOS and the identified PCOS crucial proteins. 
Fig. 5 The figure shows the interactions between Rosiglitazone and PCOS subnetwork No.3. The ellipses indicate PCOS-related proteins, the octagons show the drugs, and the Squares depict the pathways. The figure highlights the shared proteins in blue and the shared pathways in violet. The pathway protein interactions are shown by six color edges (including 1-red for Pyruvate metabolism, 2-yellow for Biosynthesis of amino acids, 3-violet for TCA cycle, 4-blue for carbon metabolism, 5-pink for metabolic pathways, and 6-orange for Glyoxylate and dicarboxylate metabolism). The yellow nodes and black edges indicate interactions among PCOSrelated proteins in subnetwork No.3 that are not shared with the drugs-related proteins

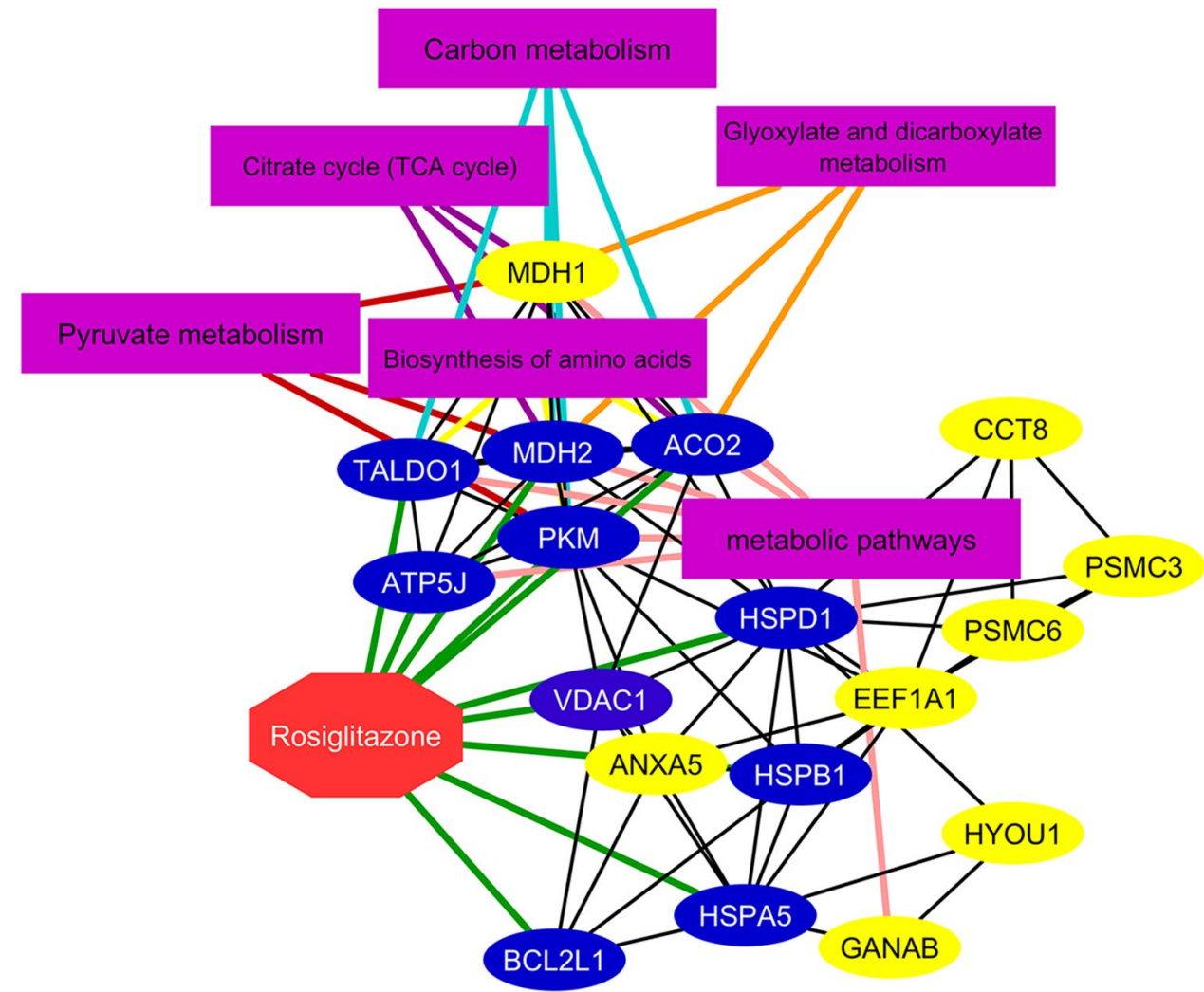

Table 4 PCOS-drug shared pathways. The shared significantly enriched pathways among PCOS, and the drugs were identified using the DAVID web tool

\begin{tabular}{lll}
\hline $\begin{array}{l}\text { PCOS sub- } \\
\text { network }\end{array}$ & PCOS-associated drugs & Shared pathway \\
\hline No.2 & $\begin{array}{l}\text { Spironolactone } \\
\text { Pioglitazone } \\
\text { Metformin }\end{array}$ & PI3K-Akt signaling pathway \\
& Letrozole \\
Rosiglitazone & \\
No.3 & $\begin{array}{l}\text { Metabolic pathways } \\
\text { Citrate cycle (TCA cycle) } \\
\end{array}$ & $\begin{array}{l}\text { Carbon metabolism } \\
\text { Pyruvate metabolism } \\
\end{array}$ \\
& & $\begin{array}{l}\text { Biosynthesis of amino acids } \\
\text { Glyoxylate and dicarboxy- } \\
\text { late metabolism }\end{array}$ \\
& & \\
& & \\
& &
\end{tabular}

\section{Discussion}

Several methods are being used to repurpose new potential drugs for the disease. Some of them are drug-based strategies, while others use disease-based strategies. Drug-based strategies use data related to molecular, chemical, pharmaceutical, and genomic information for predicting new therapeutic potentials for existing drugs [35]. However, disease-based strategies depend on phenotypic traits information, indication information, and side effects to predict therapeutic potentials for existing drugs [36]. Computational drug repositioning methods apply machine learning, network analysis, bipartile graph, clustering, and network centrality measures [37]. This study used a drug-based strategy and applied the proteomics data, network analysis, and cluster identification to predict other potential medications for PCOS treatment. We analyzed hub and bottleneck proteins in the PCOS network. Besides, we investigated the relationship between several drugs and the PCOS MCODE clusters.

Here we show that some nodes with the highest degree values (hub) play essential roles in PCOS patients' pathophysiology. (Table 2) Serum albumin (ALB) had the highest degree and was considered as a hub. According to one previous study, Serum albumin (ALB) was down-regulated in the ovaries of PCOS patients [38]. Albumin is a globular protein that binds to various bioactive molecules, including water, $\mathrm{Ca} 2+, \mathrm{Na}+, \mathrm{K}+$, fatty acids, and hormones [39]. In Buffalo, albumin enhances the maturation and fertilization rate of oocytes and improves the grade of COCs [40]. Fibronectins (FN1, FGA, and FGG) were another group of proteins with a high degree, which were down-regulated in PCOS patients' follicular fluid. They are implicated in integrin-mediated cell adhesion, assembly of the extracellular matrix, and multi-modular protein structures [41]. It 
Table 5 PCOS-drug shared biological processes. The shared significantly enriched biological processes between PCOS and the drugs were identified using the DAVID web tool

\begin{tabular}{|c|c|c|c|c|c|}
\hline $\begin{array}{l}\text { Subnet- } \\
\text { work } \\
\text { number }\end{array}$ & Drugs & Biological process & $P$-value & Shared proteins & GO ID \\
\hline \multirow[t]{3}{*}{ No.2 } & \multirow[t]{3}{*}{ Spironolactone } & secretion & $2.61 \mathrm{E}-06$ & AGT, VEGFA, TGFB1 & GO:0,046,903 \\
\hline & & single-organism transport & $3.81 \mathrm{E}-05$ & AGT, VEGFA, TGFB1 & GO:0,044,765 \\
\hline & & single-organism localization & $6.65 \mathrm{E}-05$ & AGT, VEGFA, TGFB1 & GO: $1,902,578$ \\
\hline \multirow[t]{3}{*}{ No.2 } & \multirow[t]{3}{*}{ Pioglitazone } & platelet degranulation & $4.42 \mathrm{E}-10$ & VEGFA, TGFB1, EGF & GO:0,002,576 \\
\hline & & regulated exocytosis & $1.67 \mathrm{E}-07$ & VEGFA, TGFB1, EGF & GO:0,045,055 \\
\hline & & secretion by cell & 9.91E-07 & VEGFA, TGFB1, EGF, AGT & GO:0,032,940 \\
\hline \multirow[t]{3}{*}{ No.2 } & \multirow[t]{3}{*}{ Metformin } & negative regulation of the cellular process & $3.56 \mathrm{E}-04$ & AMBP, RBP4, AGT, VEGFA, TGFB1 & GO:0,048,523 \\
\hline & & response to stress & 5.37E-04 & AMBP, AGT, VEGFA, TGFB1 & GO:0,006,950 \\
\hline & & $\begin{array}{l}\text { negative regulation of the biological } \\
\text { process }\end{array}$ & 7.04E-04 & AMBP, RBP4, AGT, VEGFA, TGFB1 & GO:0,048,519 \\
\hline \multirow[t]{3}{*}{ No.2 } & \multirow[t]{3}{*}{ Letrozole } & tube development & $1.54 \mathrm{E}-04$ & VEGFA, TGFB1 & GO:0,035,295 \\
\hline & & response to stress & 5.37E-04 & VEGFA, TGFB1 & GO:0,006,950 \\
\hline & & response to oxygen-containing compound & 0.001629 & TGFB1 & GO: $1,901,700$ \\
\hline \multirow[t]{3}{*}{ No.3 } & \multirow[t]{3}{*}{ Rosiglitazone } & response to organic substance & 7.02E-04 & PKM, HSPB1, HSPD1, BCL2L1, HSPA5 & GO:0,010,033 \\
\hline & & $\begin{array}{l}\text { organonitrogen compound metabolic } \\
\text { process }\end{array}$ & 7.39E-04 & PKM, HSPB1, MDH2, ATP5J, MDH1 & GO: $1,901,564$ \\
\hline & & small-molecule metabolic process & 0.001909 & $\begin{array}{l}\text { PKM, TALDO1, ACO2, MDH2, ATP5J, } \\
\text { MDH1 }\end{array}$ & GO:0,044,281 \\
\hline
\end{tabular}

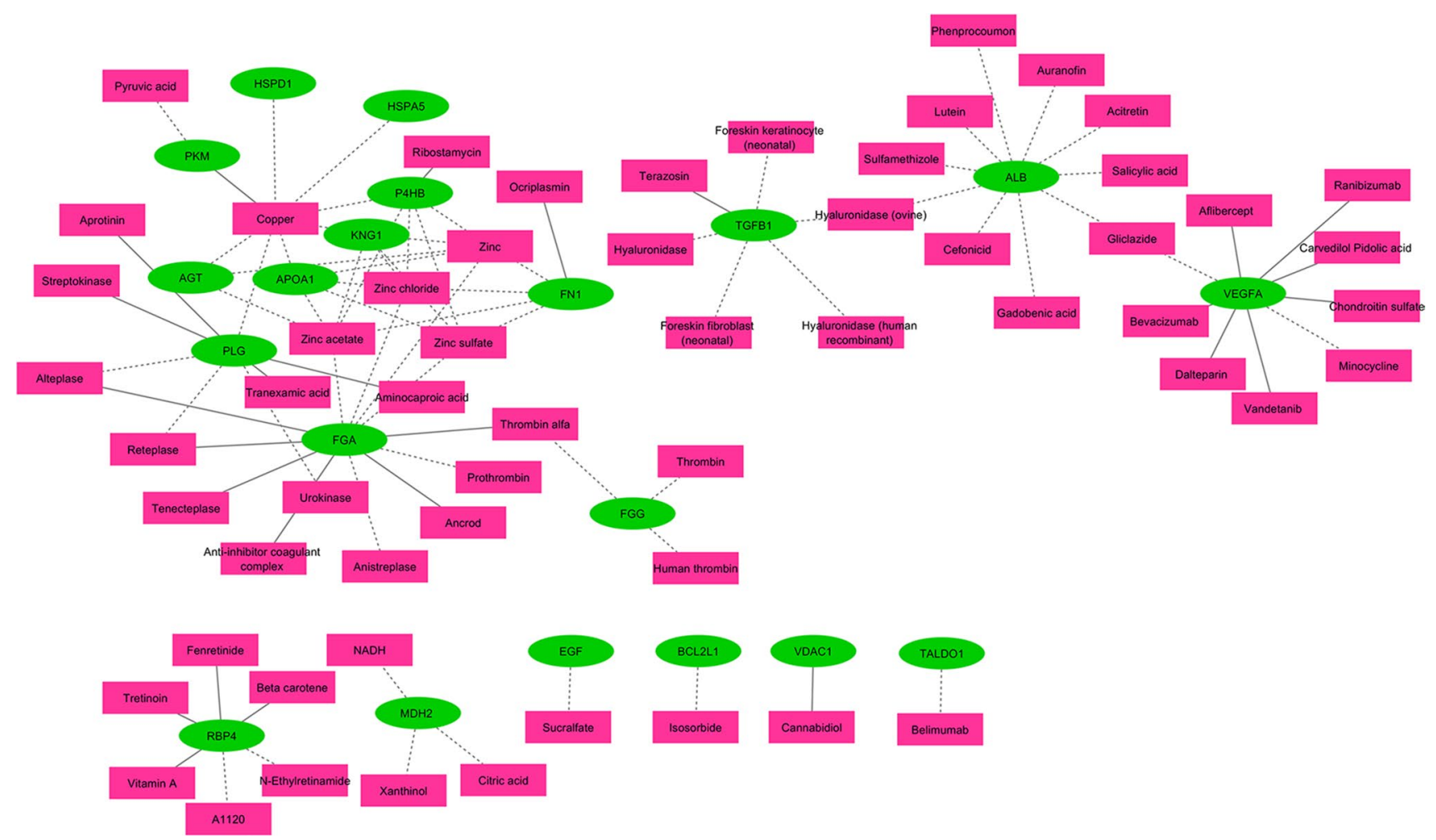

Fig. 6 Drug-protein interactions. Green nodes depict the proteins, and pink nodes represent the drugs. The dashed lines (--- -) and the continuous lines ( ) show direct and indirect interactions, respectively 
has been reported that Fibronectin affects cumulus expansion and polar body extrusion [42], so it regulates cell adhesion and plays a role in oocyte maturation. Plasminogen (PLG) and Kininogen-1 were the other two proteins with high degrees that were down-regulated and up-regulated in PCOS patients, respectively $[41,43]$. The roles of some hub proteins in the PCOS pathogenesis are already verified. We predict that further experimental studies would probably validate the part of other hubs in PCOS pathogenesis.

We investigated the significance of the network relationships among the seven US FDA-approved drugs and PCOS MCODE clusters in the second section. Five drugs were significantly related to two of the PCOS MCODE motifs based on the Chi-square/Fisher's exact test ( $p$-value $<0.05)$. The shared proteins between the drug-related proteins and PCOS clusters were identified as essential in PCOS pathogenesis. The PCOS-drug subnetwork No. 2 depicts the relationship between PCOS and metformin, letrozole, pioglitazone, and spironolactone. VEGF, EGF, TGFB1, AGT, AMBP, RBP4 proteins, and the PI3K/AKT pathway were shared (Fig. 4). VEGF and EGF are related to the PI3K/AKT pathway. They have the highest degree among the shared proteins.

The PI3K/PTEN/AKT pathway has fundamental cellular functions including, growth, survival, transcription, translation, and proliferation [44]. It plays crucial regulatory roles in ovarian function, such as activation and survival of primordial follicles. It also affects oocyte maturation and regulates the proliferation and differentiation of granulosa and theca cells. In 2014, Makker et al. showed that irregularities in the PI3K pathway associates with impaired follicular/ oocyte development and impaired ovulation [45]. Besides, in 2019, Tian-Yu Zhang et al. showed that exposure to Ochratoxin A impairs the proliferation and apoptosis of Granulosa cells through the PI3K/AKT pathway in a porcine model [46].

VEGF was found as a shared protein among subnetwork No. 2 and four drugs (Metformin, Letrozole, Pioglitazone, and Spironolactone). VEGF acts as an angiogenesis factor. VEGF increases in granulosa, theca, and luteal cells of PCOS patients. It also rises in their follicular fluid. Increased VEGF leads to increased blood flow, vascularization, and Ovarian Hyperstimulation Syndrome (OHSS) in PCOS patients [47]. The PI3K/AKT pathway leads to an increase in angiogenesis through an increase in VEGF. Tiazolididone drugs (TZD), including rosiglitazone, pioglitazone, and troglitazone, have been reported to inhibit VEGF-induced angiogenesis [48]. Other drugs, such as metformin, spironolactone, and letrozole, also have anti-angiogenic effects. They reduce the risk of ovarian hyperstimulation syndrome (OHSS) by lowering the VEGF level [49-51].

The other shared protein was EGF. In PCOS patients, the epidermal growth factor (EGF) increases [52]. In granulose cells, EGF inhibits estrogen synthesis and blocks antral follicle growth. It leads to follicular arrest in PCOS patients [53]. The EGF increment in women with POCS can lead to disorders in ovarian function through PI3K/ AKT/mTORC1. So, EGF, a shared protein between subnetwork No.2 and pioglitazone, is involved in the PI3K/ AKT pathway. Takata $Y$ et al. reported the pioglitazone as a specific antagonist of EGF that inhibits the EGF receptor tyrosine kinase [54]. So, pioglitazone probably reduces the activation of EGF through the inhibition of its target receptor.

TGFB was another shared protein between subnetwork No.2 and metformin, letrozole, pioglitazone, and spironolactone. This protein probably inhibits meiotic resumption in porcine cumulus-oocyte complexes [55]. Letrozole inhibits estrogen production by repressing the aromatase enzyme [56]. Meimei Liu et al. reported that metformin reduces TGF-B1 and improves chronic inflammation [57]. Yamada et al. suggested that pioglitazone can suppress the TGF-B superfamily and improve these patients [58]. Spironolactone is an anti-androgen drug that can reduce androgens and decrease TGF-B [59].

Angiotensin (AGT), the other shared protein between subnetwork No.2 and Metformin, Pioglitazone, and Spironolactone, is down-regulated in follicular fluid of PCOS patients [41]. Angiotensin II receptors are located on steroidogenic cells and are involved in synthesizing steroid hormones [60, 61]. The steroid hormones are considered as possible markers for oocyte maturation and cumulus expansion [62]. Wei Zhang et al. showed that pioglitazone increases the expression of Angiotensin-Converting Enzyme 2 (ACE2) [63]. Spironolactone is an antagonist for aldosterone and androgen receptors. It can be used in the treatment of PCOS [64]. The $\alpha$-1-microglobulin/bikunin precursor (AMBP), the other shared protein between subnetwork No.2 and metformin, is a component of the inter- $\alpha$-trypsin inhibitor chain and acts in the Cumulus Oocyte Complex (COC) matrix formation and its expansion [65]. Down-regulation of AMBP and TNFAIP6 (TNF Alpha Induced Protein 6) disrupts the matrix organization and expansion of the COCs in PCOS [66].

Retinol-binding protein 4 (RBP4), the shared protein between subnetwork No.2 and metformin, is expressed in theca cells. High levels of this protein were observed in fluids of follicular cysts [67]. The RBP4 level is also higher in fluids of large follicles in comparison with fluids of small follicles. RBP4 acts in retinol transport and accumulation in follicular fluids of the dominant follicles [68]. It is involved in retinoid homeostasis and the physiological function of the ovaries. Regulation of RBP4 expression during follicle development is essential for altering the ovary's retinoid levels during follicle development [69]. These correlations suggest that AGT, AMBP, and RBP4 are affected by metformin, but the exact mechanisms by which metformin affects them 
appear to be unknown. Discovering the action mechanism of metformin and these proteins may also better explain these proteins' role in the disease phenotype.

Rosiglitazone was significantly related to PCOS subnetwork No.3. Their shared proteins included HSPB1, HSPD1, ACO2, TALDO1, VDAC1, MDH2, HSPA5, ATP5J, BCL2L1, and PKM. Pyruvate metabolism, Biosynthesis of amino acids, Tricarboxylic acid cycle (Krebs cycle), Carbon metabolism, Glyoxylate, Dicarboxylate metabolism, and Metabolic pathways were the other significant shared biochemical pathway terms between PCOS and rosiglitazone. These findings suggest that improving the phenotype of the disease in PCOS patients by rosiglitazone is also likely by regulating metabolic pathways.

Zinc and copper were identified as two repurposed medications. Interestingly, in women with PCOS syndrome, zinc levels are reduced. The zinc level is introduced as one of the possible causes of insulin resistance in these patients. The use of zinc compounds can improve their insulin resistance and lipid metabolism [70]. Some antioxidant enzymes require copper and zinc for having a proper function in oocyte maturation, ovulation, and fertilization [71]. Zinc plays a role in homeostasis, cell growth, hormone release, immunological responses, and biological reproduction. It also protects cells against reactive oxygen species [72].

Reteplase and alteplase were two other medications interacting with FGA and PLG. They are novel recombinant plasminogen activators that cleavage the Arg-Val bound of endogenous plasminogen to generate plasmin [73, 74]. An increase of plasmin in the ovary can inhibit follicular rupture and improve ovulation [75]. Reteplase affects the lytic system and is an agent that contributes to the moderating of the lytic system by increasing some specific Fibrins. [76]. Gliclazide was another repurposed drug affecting VEGF and ALB. Gliclazide inhibits neovascularization through the down-regulation of VEGF. It also can suppress oxidative stress [77]. Therefore, gliclazide can be recommended as a novel therapeutic strategy in the treatment pathophysiology of PCOS. Hyaluronidase was also another identified drug that interacted with ALB and TGFB1 proteins. Hyaluronidase is reported to disrupt the extracellular matrix of oocytes and improve embryo development [78]. Previous studies have shown that removing oocyte cumulus cells (before inoculation) increases oocyte fertilization potential [79].

This study constructed and topologically analyzed the PCOS protein-protein interaction network and identified PCOS network hub/bottleneck proteins. Besides, we identified the critical proteins interacting with drugs currently used to improve ovulation in PCOS patients based on the significant relations between PCOS clusters and drugrelated proteins using Fischer exact test analysis (AGT, PKM, HSPD1, HSPA5, P4HB, KNG1, APOA1, PLG, FN1, FGA, FGG, VEGF, ALB, TGFB1, BCL2L1, PKM, MDH2,
VDAC1, TALDO1, RBP4, EGF). We attempted to predict new medications targeting the critical or similar identified proteins using the DrugBank database. Hao Huang et al. also reported a new computational approach to determine PPDT-Modules and PCOS potential drug targets in protein-protein interaction networks (PPIN). In their study, one PPDT-Module and 21 PCOS drug targets were identified, which 42 drugs targeting 13 PCOS drug targets (ESR1, RXRA, NCOA1, ESR2, THRB, RARA, PPARA, PPARG, PGR, ESRRG, RXRB, RARG, and VDR) were reported to be previously investigated experimentally [80]. Yu Wang et al. also attempted to predict candidate target proteins related to PCOS and its known targets for clinical drugs and suggested some potential candidate targets, including ESR1, PGR, AR, AKR1C3, INSR, THRB, PTPN1, DPP4, NR3C1, HSD11B1, and METAP2 for berberine and other drugs related to PCOS. They also constructed a drug-target network and analyzed it [81].

In brief, some results of this in-silico analysis in identifying the crucial molecular mechanisms underlying the PCOS pathogenesis have been verified by other previous experimental studies. Some other findings within this textmined in-silico prediction demand further study in vitro and in vivo. In this study, we also repurposed some new drug candidates for PCOS treatment (Table 6). Herein, we discuss that some of our drug candidates have already shown beneficial effects in improving PCOS patients' symptoms. Therefore, we recommend other repurposed drugs to be experimentally investigated in vitro and in vivo for their possible healing effect in PCOS.

\section{Conclusions}

In this study, the significant relationships between a PPI MCODE cluster and most investigated drugs revealed the pivotal role of the PI3K/AKT pathway in PCOS pathogenesis. The PI3K/AKT pathway probably intermediates PCOS pathogenesis through cell proliferation, survival, growth, metabolism, and angiogenesis. Therefore, it may contribute to the PCOS pathogenesis in ovulation and oocyte maturation through the survival of primordial follicles, proliferation/differentiation of granulosa/theca cells, and oocyte maintenance/activation. Our study also predicted some other probable biological processes and pathways underlying PCOS, including the metabolic pathways and the Cell death pathway. Various biological processes were involved in the PCOS pathogenesis, including 1-secretion, 2-response to stress, 3-regulated exocytosis, and 4-platelet degranulation. These probably play vital roles in PCOS molecular pathogenesis too. Some of our in-silico prediction results were recently verified by other experimental studies, while others remain to be further 
Table 6 The table represents the repurposed FDA-approved medications for PCOS and the identified PCOS crucial proteins. The repurposed medicines are sorted by the number of proteins interacting with them in the constructed drug-protein interaction network

\begin{tabular}{llll}
\hline DrugBank ID & repurposed FDA-approved Drugs & $\begin{array}{l}\text { Number of proteins interact- } \\
\text { ing with drug }\end{array}$ & Proteins \\
\hline DB09130 & Copper & 8 & $\begin{array}{c}\text { AGT, PKM, HSPD1, HSPA5, P4HB, } \\
\text { KNG1, APOA1, PLG }\end{array}$ \\
DB14487 & Zinc acetate & 6 & P4HB, KNG1, FN1, APOA1, AGT, FGA \\
DB01593 & Zinc & 6 & AGT, APOA1, KNG1, P4HB, FN1, FGA \\
DB09322 & Zinc sulfate & 5 & P4HB, FN1, FGA, KNG1, APOA1 \\
DB14533 & Zinc chloride & 5 & KNG1, P4HB, FN1, FGA, APOA1 \\
DB11572 & Thrombin alfa & 2 & FGG, FGA \\
DB00015 & Reteplase & 2 & FGA, PLG \\
DB00009 & Alteplase & 2 & FGA, PLG \\
DB01120 & Gliclazide & 2 & VEGF, ALB \\
DB00070 & Hyaluronidase (ovine) & 2 & ALB, TGFB1 \\
\hline
\end{tabular}

investigated in vitro and in vivo. In PCOS, treatments had better be selected based on specific patient manifestations and be individualized. Personalized medicine requires in-depth knowledge of the various possible molecular pathologies of the disease. Overall, using a systems biology approach could help predict a more in-depth insight into the molecular pathology behind the PCOS mechanism that could influence the future design of prophylactic and therapeutic drugs and fill our knowledge gap toward personalized medicine. In this study, we identified the crucial molecules underlying PCOS pathogenesis using a systems biology approach. We then repurposed some new candidate drugs for PCOS based on the essential molecules. We recommend them to be investigated in vitro and in vivo for their possible role in healing PCOS.

Supplementary Information The online version contains supplementary material available at https://doi.org/10.1007/s40199-021-00413-9.

Acknowledgements We thank the services provided by the biotechnology department of the School of Advanced Technologies in Medicine, Shahid Beheshti University of Medical Sciences.

Funding This research received no specific grant from any funding agency in the public, commercial, or not-for-profit sectors.

Availability of data and material Readers may have access to the raw data, details of the analyzed data, and issues in the represented supplementary files.

Code availability The software used was free (Cytoscape).

\section{Declarations}

Compliance with ethical standards Not applicable.

Conflicts of interest/Competing interests The authors declare that they have no conflict of interest.
Ethics approval Not applicable.

Consent to participate Not applicable.

Consent for publication Not applicable.

\section{References}

1. Franks S. Polycystic ovary syndrome. Engl J Med. 1995;333:853-61.

2. Younas K, Quintela M, Thomas S, Garcia-Parra J, Blake L, Whiteland H, Bunkheila A, Francis LW, Margarit L, Gonzalez D. Delayed endometrial decidualisation in polycystic ovary syndrome; the role of AR-MAGEA11. J Mol Med. 2019;97:1315-27.

3. Jakimiuk AJ, Weitsman SR, Brzechffa PR, Magoffin DA. Aromatase mRNA expression in individual follicles from polycystic ovaries. Mol Hum Reprod. 1998;4:1-8.

4. Aubuchon M, Legro RS. Polycystic ovary syndrome: current infertility management. Best Pract Res Clin Obstet Gynaecol. 2011;54:675-84.

5. Gharani N, Waterworth DM, Batty S, White D, Gilling-Smith C, Conway GS, McCarthy M, Franks S, Williamson R. Association of the steroid synthesis gene CYP11a with polycystic ovary syndrome and hyperandrogenism. Hum Mol Genet. 1997;6:397-402.

6. Carey AH, Waterworth D, Patel K, White D, Little J, Novelli P, Franks S, Williamson R. Polycystic ovaries and premature male pattern baldness are associated with one allele of the steroid metabolism gene CYP17. Hum Mol Genet. 1994;3:1873-6.

7. Dunaif A, Segal KR, Shelley DR, Green G, Dobrjansky A, Licholai T. Evidence for distinctive and intrinsic defects in insulin action in polycystic ovary syndrome. Diabetes. 1992;41:1257-66.

8. Radosh L. Drug treatments for polycystic ovary syndrome. Am Fam Physician. 2009;79:671-6.

9. Si Zheng SD. Meng Wu, Jiao Li, Zhiyong Lu Text Mining for Drug Discovery. Methods Mol Biol. 2019;1939:231-52.

10. Pletscher-Frankild $S$, Pallejà $A$, Tsafou K, Binder JX, Jensen LJ. DISEASES: Text mining and data integration of disease-gene associations. Methods. 2015;74:83-9.

11. Ramly B, Afiqah-Aleng N, Mohamed-Hussein Z-A. Protein-protein interaction network analysis reveals several diseases highly associated with polycystic ovarian syndrome. Int J Mol Sci. 2019;20:2959. 
12. von Eichborn J, Dunkel M, Gohlke BO, Preissner SC, Hoffmann MF, Bauer JM, Armstrong JD, Schaefer MH, Andrade-Navarro MA, Le Novere N. SynSysNet: integration of experimental data on synaptic protein-protein interactions with drug-target relations. Nucleic acids Res. 2012;41:D834-40.

13. Ma J, Wang J, Ghoraie LS, Men X, Haibe-Kains B, Dai P. A comparative study of cluster detection algorithms in protein-protein interaction for drug target discovery and drug repurposing. Front Pharmacol. 2019;10:109.

14. Macalino SJY, Basith S, Clavio NAB, Chang H, Kang S, Choi $\mathrm{S}$. Evolution of in silico strategies for protein-protein interaction drug discovery. Molecules. 2018;23:1963.

15. Murakami Y, Tripathi LP, Prathipati P, Mizuguchi K. Network analysis and in silico prediction of protein-protein interactions with applications in drug discovery. Curr Opin Struct Biol. 2017;44:134-42.

16. Legro RS: Evaluation and treatment of polycystic ovary syndrome. In Endotext [Internet]. MDText. com, Inc.; 2017

17. Welter D, MacArthur J, Morales J, Burdett T, Hall P, Junkins H, Klemm A, Flicek P, Manolio T, Hindorff L. The NHGRI GWAS Catalog, a curated resource of SNP-trait associations. Nucleic acids Res. 2014;42:D1001-6.

18. Espe S. MalaCards: the human disease database. JMLA. 2018;106:140.

19. Li MJ, Liu Z, Wang P, Wong MP, Nelson MR. Kocher J-PA, Yeager M, Sham PC, Chanock SJ, Xia Z, GWASdb v2: an update database for human genetic variants identified by genome-wide association studies. Nucleic acids Res. 2016;44:D869-76.

20. Amberger JS, Bocchini CA, Schiettecatte F, Scott AF, Hamosh A, OMIM. org: Online Mendelian Inheritance in Man (OMIM®), an online catalog of human genes and genetic disorders, Nucleic Acids Res, 43:D789-D798,2015.

21. Peng K, Xu W, Zheng J, Huang K, Wang H, Tong J, Lin Z, Liu J, Cheng W, Fu D. The Disease and Gene Annotations (DGA): an annotation resource for human disease. Nucleic acids Res. 2012;41:D553-60.

22. Kahraman A, Avramov A, Nashev LG, Popov D, Ternes R, Pohlenz H-D, Weiss B. PhenomicDB: a multi-species genotype/ phenotype database for comparative phenomics. Bioinformatics. 2005;21:418-20.

23. Piñero J, Bravo À, Queralt-Rosinach N, Gutiérrez-Sacristán A, Deu-Pons J, Centeno E, García-García J, Sanz F, Furlong LI, DisGeNET: a comprehensive platform integrating information on human disease-associated genes and variants, Nucleic acids Res:gkw943,2016.

24. Stenson PD, Mort M, Ball EV, Shaw K, Phillips AD, Cooper DN. The Human Gene Mutation Database: building a comprehensive mutation repository for clinical and molecular genetics, diagnostic testing and personalized genomic medicine. Hum Genet. 2014;133:1-9.

25. Davis AP, Grondin CJ, Johnson RJ, Sciaky D, McMorran R, Wiegers J, Wiegers TC, Mattingly CJ. The comparative toxicogenomics database: update 2019. Nucleic acids Res. 2019;47:D948-54.

26. Kuhn M, von Mering C, Campillos M, Jensen LJ, Bork P. STITCH: interaction networks of chemicals and proteins. Nucleic acids Res. 2007;36:D684-8.

27. Bader GD, Hogue CW. An automated method for finding molecular complexes in large protein interaction networks. BMC Bioinformatics. 2003;4:2.

28. Rana R, Singhal R. Chi-square test and its application in hypothesis testing. J Pract Cardio Sci. 2015;1:69.

29. Fisher RA. On the interpretation of $\chi 2$ from contingency tables, and the calculation of P. J R Stat Soc Series B Stat Methodol. 1922;85:87-94.

30. Fuentealba M, Dönertaş HM, Williams R, Labbadia J, Thornton JM, Partridge L, Using the drug-protein interactome to identify anti-ageing compounds for humans, PLoS Comput Biol, 15:e1006639,2019.

31. Takahashi Y, Terada T, Muto Y. Systems Level Analysis and Identification of Pathways and Key Genes Associated with Delirium. Genes. 2020;11:1225.

32. Sherman BT, Lempicki RA. Systematic and integrative analysis of large gene lists using DAVID bioinformatics resources. Nat Protoc. 2009;4:44.

33. Kanehisa M, Furumichi M, Tanabe M, Sato Y, Morishima K. KEGG: new perspectives on genomes, pathways, diseases and drugs. Nucleic acids Res. 2017;45:D353-61.

34. Safaei A, Tavirani MR, Oskouei AA, Azodi MZ, Mohebbi SR, Nikzamir AR. Protein-protein interaction network analysis of cirrhosis liver disease. GASTROENTEROL HEPATOL BED BENCH. 2016;9:114.

35. Dudley JT, Deshpande T, Butte AJ. Exploiting drug-disease relationships for computational drug repositioning. Brief Bioinform. 2011;12:303-11.

36. Ashburn TT, Thor KB. Drug repositioning: identifying and developing new uses for existing drugs. Nat Rev Drug Discovery. 2004;3:673-83.

37. Jarada TN, Rokne JG, Alhajj R. A review of computational drug repositioning: strategies, approaches, opportunities, challenges, and directions. Journal of Cheminformatics. 2020;12:1-23.

38. Choi Y, Kim H-R, Lim EJ, Park M, Yoon JA, Kim YS, Kim EK, Shin JE, Kim JH, Kwon H, Integrative analyses of uterine transcriptome and microRNAome reveal compromised LIFSTAT3 signaling and progesterone response in the endometrium of patients with recurrent/repeated implantation failure (RIF), PloS one, 11:e0157696,2016.

39. Lu J, Stewart AJ, Sadler PJ, Pinheiro TJ, Blindauer CA: Albumin as a zinc carrier: properties of its high-affinity zinc-binding site. Portland Press Ltd.; 2008.

40. Rahman A, Khandoker M, Asad L, Saha S, Paul R, Debnath S, In vitro maturation and fertilization of buffalo oocytes cultured in media supplemented with bovine serum albumin,2015.

41. Ambekar AS, Kelkar DS, Pinto SM, Sharma R, Hinduja I, Zaveri K, Pandey A, Prasad TK, Gowda H, Mukherjee S. Proteomics of follicular fluid from women with polycystic ovary syndrome suggests molecular defects in follicular development. J Clin Endocrinol Metab. 2015;100:744-53.

42. Pandey A, Gupta N, Gupta S. Improvement of in vitro oocyte maturation with lectin supplementation and expression analysis of Cx43, GDF-9, FGF-4 and Fibronectin mRNA transcripts in Buffalo (Bubalus bubalis). J Assist Reprod Genet. 2009;26:365-71.

43. Wong MK-S, Takei Y, Lack of plasma kallikrein-kinin system cascade in teleosts, PloS one, 8,2013.

44. Wu Y, Li P, Zhang D, Sun Y. Metformin and pioglitazone combination therapy ameliorate polycystic ovary syndrome through AMPK/PI3K/JNK pathway. Exp Ther Med. 2018;15:2120-7.

45. Makker A, Goel MM, Mahdi AA. PI3K/PTEN/Akt and TSC/ mTOR signaling pathways, ovarian dysfunction, and infertility: an update. J Mol Endocrinol. 2014;53:R103-18.

46. Zhang T-Y, Sun X-F, Li L, Ma J-M, Zhang R-Q, Li N, Liu $\mathrm{X}-\mathrm{L}$, Dyce PW, Shen W. Ochratoxin A exposure impairs porcine granulosa cell growth via the PI3K/AKT signaling pathway. J Agric Food Chem. 2019;67:2679-90.

47. Ferrara N, Frantz G, LeCouter J, Dillard-Telm L, Pham T, Draksharapu A, Giordano T, Peale F. Differential expression of the angiogenic factor genes vascular endothelial growth factor (VEGF) and endocrine gland-derived VEGF in normal and polycystic human ovaries. AM J PATHOL. 2003;162:1881-93.

48. Panigrahy D, Singer S, Shen LQ, Butterfield CE, Freedman DA, Chen EJ, Moses MA, Kilroy S, Duensing S, Fletcher C. PPAR $\gamma$ 
ligands inhibit primary tumor growth and metastasis by inhibiting angiogenesis. J Clin Invest. 2002;110:923-32.

49. Khattab S, Fotouh IA, Mohesn IA, Metwally M, Moaz M. Use of metformin for prevention of ovarian hyperstimulation syndrome: a novel approach. Reprod Biomed Online. 2006;13:194-7.

50. Hsu S-J, Wang S-S, Huo T-I, Lee F-Y, Huang H-C, Chang C-C, Hsin I-F, Ho H-L, Lin H-C, Lee S-D. The Impact of Spironolactone on the Severity of Portal-Systemic Collaterals and Hepatic Encephalopathy in Cirrhotic Rats. J Pharmacol Exp Ther. 2015;355:117-24.

51. He Q, Liang L, Zhang C, Li H, Ge Z, Wang L, Cui S. Effects of different doses of letrozole on the incidence of early-onset ovarian hyperstimulation syndrome after oocyte retrieval. SYST BIOL REPROD MED. 2014;60:355-60.

52. Dai G, Lu G. Different protein expression patterns associated with polycystic ovary syndrome in human follicular fluid during controlled ovarian hyperstimulation. Reprod Fertil Dev. 2012;24:893-904.

53. Giovanni Artini P, Monteleone P, Parisen Toldin MR, Matteucci C, Ruggiero M, Cela V, Genazzani AR. Growth factors and folliculogenesis in polycystic ovary patients. Expert Rev Endocrinol Metab. 2007;2:215-23.

54. Takata Y, Imamura T, Yang GH, Takada Y, Sawa T, Morioka H, Kobayashi M. Pioglitazone attenuates the inhibitory effect of phorbol ester on epidermal growth factor receptor autophosphorylation and tyrosine kinase activity. Biochim Biophys Acta. 1996;1312:68-72.

55. Coskun S, Lin YC. Effects of transforming growth factors and activin-A on in vitro porcine oocyte maturation. Mol Reprod Dev. 1994;38:153-9.

56. Rajan RK, Balaji B. Soy isoflavones exert beneficial effects on letrozole-induced rat polycystic ovary syndrome (PCOS) model through anti-androgenic mechanism. Pharm Biol. 2017;55:242-51

57. Liu M, Gao J, Zhang Y, Li P, Wang H, Ren X, Li C. Serum levels of TSP- $1, \mathrm{NF}-\mathrm{\kappa B}$ and TGF- $\beta 1$ in polycystic ovarian syndrome (PCOS) patients in northern China suggest PCOS is associated with chronic inflammation. J Clin Endocrinol Metab. 2015;83:913-22.

58. Yamada-Nomoto K, Yoshino O, Akiyama I, Iwase A, Ono Y, Nakamura T, Harada M, Nakashima A, Shima T, Ushijima A, PAI-1 in granulosa cells is suppressed directly by statin and indirectly by suppressing TGF- $\beta$ and TNF- $\alpha$ in mononuclear cells by insulin-sensitizing drugs, Am J Reprod Immunol, 78:e12669,2017.

59. Homburg R: Polycystic ovary syndrome in adolescence. In Abnormalities in Puberty. Volume 8: Karger Publishers; 2005: 137-149

60. Yoshimura Y. The ovarian renin-angiotensin system in reproductive physiology. Front Endocrinol. 1997;18:247-91.

61. van Sande ME, Scharpé SL, Neels HM, Van Camp KO. Distribution of angiotensin converting enzyme in human tissues. Clin Chim Acta. 1985;147:255-60.

62. Wang HF, Isobe N, Kumamoto K, Yamashiro H, Yamashita Y, Terada T. Studies of the role of steroid hormone in the regulation of oocyte maturation in cattle. Reprod Biol Endocrinol. 2006;4:4.

63. Zhang W, Xu Y-Z, Liu B, Wu R, Yang Y-Y, Xiao X-Q, Zhang $X$, Pioglitazone up-regulates angiotensin converting enzyme 2 expression in insulin-sensitive tissues in rats with high-fat dietinduced nonalcoholic steatohepatitis, Sci World J, 2014,2014.

64. Armanini D, Andrisani A, Bordin L, Sabbadin C: Spironolactone in the treatment of polycystic ovary syndrome. Taylor \& Francis; 2016.

65. Zhuo L, Yoneda M, Zhao M, Yingsung W, Yoshida N, Kitagawa Y, Kawamura K, Suzuki T, Kimata K. Defect in SHAP-Hyaluronan Complex Causes Severe Female Infertility A STUDY BY
INACTIVATION OF THE BIKUNIN GENE IN MICE. J Biol Chem. 2001;276:7693-6.

66. Wathlet S, Adriaenssens T, Segers I, Verheyen G, Van de Velde H, Coucke W, El Ron R, Devroey P, Smitz J. Cumulus cell gene expression predicts better cleavage-stage embryo or blastocyst development and pregnancy for ICSI patients. Hum Reprod. 2011;26:1035-51.

67. Sun YL, Ping ZG, Li CJ, Sun YF, Yi KL, Chen L, Li XY, Wang $\mathrm{XL}$, Zhou X. Comparative proteomic analysis of follicular fluids from normal and cystic follicles in sows. Reprod Domest Anim. 2011;46:889-95.

68. Brown JA, Eberhardt DM, Schrick FN, Roberts MP, Godkin JD. Expression of retinol-binding protein and cellular retinol-binding protein in the bovine ovary. Mol Reprod Dev. 2003;64:261-9.

69. Jiang Y, Zhao Y, Chen S, Chen L, Li C, Zhou X. Regulation by FSH of the dynamic expression of retinol-binding protein 4 in the mouse ovary. Reprod Biol Endocrinol. 2018;16:25.

70. Günalan E, Yaba A, Yılmaz B. The effect of nutrient supplementation in the management of polycystic ovary syndrome-associated metabolic dysfunctions: A critical review. J Turk Ger Gynecol Assoc. 2018;19:220.

71. Agarwal A, Gupta S, Sikka S. The role of free radicals and antioxidants in reproduction. Curr Opin Obstet Gynecol. 2006; 18:325-32.

72. Nasiadek M, Stragierowicz J, Klimczak M, Kilanowicz A. The Role of Zinc in Selected Female Reproductive System Disorders. Nutrients. 2020;12:2464.

73. Mohammadi E, Seyedhosseini-Ghaheh H, Mahnam K, JahanianNajafabadi A, Sadeghi HMM, Reteplase: structure, function, and production, Adv Biomed Res, 8,2019.

74. Marti-Fabregas J, Borrell M, Cocho D, Belvis R, Castellanos M, Montaner J, Pagonabarraga J, Aleu A, Molina-Porcel L, DiazManera J. Hemostatic markers of recanalization in patients with ischemic stroke treated with rt-PA. Neurology. 2005;65:366-70.

75. Atiomo WU, Bates SA, Condon JE, Shaw S, West JH, Prentice AG. The plasminogen activator system in women with polycystic ovary syndrome. Fertil Steril. 1998;69:236-41.

76. Meierhenrich R, Carlsson J, Seifried E, Pfarr E, Smolarz A, Neuhaus K-L, Tebbe U. Effect of reteplase on hemostasis variables: analysis of fibrin specifity, relation to bleeding complications and coronary patency. Int J Cardiol. 1998;65:57-63.

77. Kimura T, Takagi H, Suzuma K, Kita M, Watanabe D, Yoshimura N. Comparisons between the beneficial effects of different sulphonylurea treatments on ischemia-induced retinal neovascularization. Free Radic Biol Med. 2007;43:454-61.

78. de Moura BR, Gurgel MC, Machado SP, Marques PA, Rolim JR, de Lima MC, Salgueiro LL. Low concentration of hyaluronidase for oocyte denudation can improve fertilization rates and embryo quality. JBRA Assist Reprod. 2017;21:27.

79. Lavy G, Boyers SP, DeCherney AH. Hyaluronidase removal of the cumulus oophorus increases in vitro fertilization. J Vitro Fert Embryo Transfer. 1988;5:257-60.

80. Huang H, He Y, Li W, Wei W, Li Y, Xie R, Guo S, Wang Y, Jiang $\mathrm{J}$, Chen B. Identification of polycystic ovary syndrome potential drug targets based on pathobiological similarity in the proteinprotein interaction network. Oncotarget. 2016;7:37906.

81. Wang Y, Fu X, Xu J, Wang Q, Kuang H. Systems pharmacology to investigate the interaction of berberine and other drugs in treating polycystic ovary syndrome. Sci Rep. 2016;6:1-10.

Publisher's Note Springer Nature remains neutral with regard to jurisdictional claims in published maps and institutional affiliations. 